Research

Open Access

\title{
Changes in serum creatinine in the first 24 hours after cardiac arrest indicate prognosis: an observational cohort study
}

\author{
Dietrich Hasper ${ }^{1}$, Stephan von Haehling ${ }^{2}$, Christian Storm ${ }^{1}$, Achim Jörres ${ }^{1}$ and Joerg C Schefold ${ }^{1}$
}

1Charité-Universitätsmedizin Berlin, Campus Virchow-Klinikum, Department of Nephrology and Medical Intensive Care, Augustenburger Platz 1,
13353 Berlin, Germany
${ }^{2}$ Charité-Universitätsmedizin Berlin, Campus Virchow-Klinikum, Department of Clinical Cardiology, Augustenburger Platz 1, 13353 Berlin, Germany

Corresponding author: Dietrich Hasper, dietrich.hasper@charite.de

Received: 16 Jul 2009 Revisions requested: 2 Sep 2009 Revisions received: 22 Sep 2009 Accepted: 29 Oct 2009 Published: 29 Oct 2009

Critical Care 2009, 13:R168 (doi:10.1186/cc8144)

This article is online at: http://ccforum.com/content/13/5/R168

(C) 2009 Hasper et al.; licensee BioMed Central Ltd.

This is an open access article distributed under the terms of the Creative Commons Attribution License (http://creativecommons.org/licenses/by/2.0), which permits unrestricted use, distribution, and reproduction in any medium, provided the original work is properly cited.

\begin{abstract}
Introduction As patients after cardiac arrest suffer from the consequences of global ischemia reperfusion, we aimed to establish the incidence of acute kidney injury (AKI) in these patients, and to investigate its possible association to severe hypoxic brain damage.

Methods One hundred and seventy-one patients (135 male, mean age 61.6 +/- 15.0 years) after cardiac arrest were included in an observational cohort study. Serum creatinine was determined at admission and 24, 48 and 72 hours thereafter. Serum levels of neuron-specific enolase (NSE) were measured 72 hours after admission as a marker of hypoxic brain damage. Clinical outcome was assessed at intensive care unit (ICU) discharge using the Pittsburgh cerebral performance category (CPC).

Results AKI as defined by AKI Network criteria occurred in 49\% of the study patients. Patients with an unfavourable prognosis (CPC 3-5) were affected significantly more frequently $(P=$ $0.013)$. Whilst serum creatinine levels decreased in patients with good neurological outcome (CPC 1 or 2) over the ensuing 48 hours, it increased in patients with unfavourable outcome

(CPC 3-5). ROC analysis identified DeltaCrea24 <-0.19 mg/dl as the value for prediction with the highest accuracy. The odds ratio for an unfavourable outcome was 3.81 (95\% Cl 1.98-7.33, $P=0.0001)$ in cases of unchanged or increased creatinine levels after 24 hours compared to those whose creatinine levels decreased during the first 24 hours. NSE levels were found to correlate with the change in serum creatinine in the first 24 hours both in simple and multivariate regression (both $r=0.24$, $P=0.002$ ).

Conclusions In this large cohort of patient after cardiac arrest, we found that AKI occurs in nearly $50 \%$ of patients when the new criteria are applied. Patients with unfavourable neurological outcome are affected more frequently. A significant association between the development of AKI and NSE levels indicating hypoxic brain damage was observed. Our data show that changes in serum creatinine may contribute to the prediction of outcome in patients with cardiac arrest. Whereas a decline in serum creatinine $(>0.2 \mathrm{mg} / \mathrm{dL})$ in the first 24 hours after cardiac arrest indicates good prognosis, the risk of unfavourable outcome is markedly elevated in patients with constant or increasing serum creatinine.
\end{abstract}

\section{Introduction}

Acute kidney injury (AKI) is a common and devastating problem in critically ill patients. Although sepsis is the most frequent cause of $\mathrm{AKI}$ in the intensive care setting, a number of other clinical conditions may induce renal failure [1]. Small changes in serum creatinine are associated with an increased mortality risk in hospitalised patients [2]. Following multiple and variable definitions of renal failure in the past, the Acute
Kidney Injury Network has recently proposed uniform standards for diagnosing and classifying AKI [3]. This set of criteria has proven to be a valuable tool in various clinical situations [47]. Besides an improved definition of renal failure, much scientific effort has focused on the identification of the complex pathobiology of AKI in order to define new therapeutic targets. In recent years, animal models have mostly focused on renal ischemia and reperfusion (e.g. renal vascular cross clamp or 
high-dose norepinephrine infusion) [8]. Based on these studies renal ischemia/reperfusion is regarded as being a major contributor to the development of $\mathrm{AKI}$ in critically ill patients [9]. However, in the majority of patients it remains unknown whether AKI is caused by systemic versus renal hypoperfusion, circulating nephrotoxins, or additional insults.

Cardiac arrest may be considered as a model of systemic ischemia/reperfusion. Patients surviving cardiac arrest suffer from global ischemia/reperfusion affecting all end organs including the brain. Hypoxic encephalopathy is arguably the most important determinant of patient outcome in this setting, and it has been demonstrated previously that long-term prognosis depends more on the degree of hypoxic brain damage than on the underlying disease [10].

The extent of hypoxic brain damage can be estimated by measurement of serum levels of the enzyme neurone-specific enolase (NSE). This enzyme is a protein contained by neurons and is released into the circulation after neuronal cell damage. Peak serum levels reflect the amount of neuronal damage and correlate with clinical outcomes [11-13]. As a consequence, NSE serum levels may indicate the degree of hypoxic burden in patients surviving cardiac arrest.

Assuming that both the brain and kidney are sensitive to ischemia, hypoxic damage should affect both organs; however, few data to this end are presently available. We therefore set out to investigate the potential relation between hypoxic encephalopathy and AKI in patients after cardiac arrest. The new criteria defining $A K I$ were applied to these patients and correlated to both NSE levels and short-term neurological outcome.

\section{Materials and methods}

The study protocol was approved by the local ethics committee on human research. All data were collected within the normal daily intensive care routine in an anonymous fashion. The institutional review board therefore waived the need for informed patient consent. In a retrospective analysis, we iden- tified a total of 195 patients who were admitted to the medical intensive care unit (ICU) of a tertiary care academic center after cardiac arrest between January 2003 and December 2007. In all patients, care was directed by critical care physicians based on standard operating procedures. Following our standard of care all patients received full ICU support over the first three days. Cardiac catheterization was performed as soon as possible when indicated. Patients admitted after December 2005 were treated with therapeutic hypothermia for 24 hours irrespective of the initial cardiac rhythm. According to our standard of treatment, neurological outcome was assessed after the third day using measures of clinical evaluation, NSE serum levels and somatosensory-evoked potentials when needed.

Seven patients died before the third day of ICU stay and were therefore excluded from further analysis. Another 12 patients were excluded due to incomplete data records and two patients because of pre-existing need for renal replacement therapy. Patients were excluded when pre-existing advanced renal disease was present. Advanced renal disease was defined as an estimated glomerular filtration rate less than 30 $\mathrm{ml} / \mathrm{min} / 1.73 \mathrm{~m}^{2}$ at ICU admission (calculated using the simplified equation derived from the 'Modification of Diet in Renal Disease'(MDRD) study) [14]. Thus, three patients in Kidney Disease Outcome Quality Initiative stages 4 and 5, indicating severe and very severe renal failure, were excluded. The remaining 171 patients entered the analysis presented here. Blood samples for determination of serum creatinine levels were drawn immediately after ICU admission and every 24 hours thereafter. The difference between admission (i.e. baseline) serum creatinine and the values after 24 and 72 hours were calculated as $\Delta$ Crea24 and $\Delta$ Crea 72 .

AKI was defined by the criteria published by Mehta and colleagues [3] using the serum creatinine at admission as baseline value (Table 1). NSE serum levels were measured 72 hours after admission with an enzyme immunoassay (Elecsys 2010, Roche Diagnostics GmbH, Mannheim, Germany).

Table 1

\section{Classification/staging system for acute kidney injury}

\begin{tabular}{|c|c|c|}
\hline Stage & Serum creatinine criteria & Urine output criteria \\
\hline 1 & $\begin{array}{l}\text { Increase in serum creatinine of more than or equal to } 0.3 \mathrm{mg} / \mathrm{dl} \text { ( } \geq \\
26.4 \mu \mathrm{mol} / \mathrm{l} \text { ) or increase to more than or equal to } 150 \% \text { to } 200 \% \text { (1.5 } \\
\text { to } 2 \text {-fold) from baseline }\end{array}$ & Less than $0.5 \mathrm{ml} / \mathrm{kg}$ per hour for more than 6 hours \\
\hline 2 & $\begin{array}{l}\text { Increase in serum creatinine to more than } 200 \% \text { to } 300 \%(>2 \text { to } 3 \text { - } \\
\text { fold) from baseline }\end{array}$ & Less than $0.5 \mathrm{ml} / \mathrm{kg}$ per hour for more than 12 hours \\
\hline 3 & $\begin{array}{l}\text { Increase in serum creatinine to more than } 300 \% \text { ( }>3 \text {-fold }) \text { from } \\
\text { baseline (or serum creatinine of more than or equal to } 4.0 \mathrm{mg} / \mathrm{dl}(\geq \\
354 \mu \mathrm{mol} / \mathrm{l}) \text { with an acute increase of at least } 0.5 \mathrm{mg} / \mathrm{dl}(44 \mu \mathrm{mol} / \mathrm{l}))\end{array}$ & Less than $0.3 \mathrm{ml} / \mathrm{kg}$ per hour for 24 hours or anuria for 12 hours \\
\hline
\end{tabular}

Classification/staging system for acute kidney injury as provided by Mehta and colleagues [3]. Individuals who receive renal replacement therapy are considered to have met the criteria for stage 3 irrespective of the stage they are in at the time of renal replacement therapy. 
Neurological outcome was assessed at the time of ICU discharge according to the Pittsburgh cerebral performance category (CPC) [15]. The classifying physician was blinded to the intention of the study. CPC 1 and 2 were classified as a favorable neurological outcome whereas CPC 3, 4 and 5 were regarded as an unfavorable outcome.

The software MedCalc ${ }^{\circledR}$ 9.3.2 (MedCalc Software, Mariakerke, Belgium) was used for statistical analysis. Continuous data are presented as median and 25 to $75 \%$ interquartile range (IQR) unless stated otherwise. Binary variables are presented as numbers and percentages. Mann-Whitney $U$ testing was performed to compare continuous data, and Fisher's exact test was used to compare proportions. Simple and multivariable regression analyses were used as appropriate. Sensitivity and specificity of $\Delta$ Crea24 to predict outcomes were determined by analysis of receiver-operator characteristics (ROC) curves. The significance level was set at $P<0.05$.

\section{Results}

\section{Study population and neurological outcomes}

Basic characteristics of the 171 cardiac arrest patients included in this study are presented in Table 2. With regards to neurological outcome, 69 patients had a favorable neurological outcome with either CPC $1(n=39,22.8 \%)$ or CPC 2 $(n=30,17.5 \%)$. Ten patients $(5.8 \%)$ had moderate (CPC 3 ) and 24 patients $(24.0 \%)$ severe neurological disability (CPC 4) at ICU discharge, and 68 patients (39.8\%) died before ICU discharge (CPC 5). As a result of neurological assessment after the third ICU day, 87 patients (51\%) had a do not resuscitate-order. Although those in the poor CPC group compared with the favorable CPC group were on average older, more likely to be female, and less likely to receive bystander cardiopulmonary resuscitation, these differences did not attain statistical significance. As expected, a favorable outcome was significantly associated with ventricular fibrillation as monitored as an initial rhythm, lower NSE serum levels and the application of therapeutic hypothermia.

\section{Course of serum creatinine}

In the overall study population a median serum creatinine at admission of $1.24 \mathrm{mg} / \mathrm{dl}$ (1.01 to $1.65 \mathrm{mg} / \mathrm{dl}$ ) was measured. Over the ensuing two days, a significant drop of serum creatinine was observed with lowest values observed at ICU discharge (Table 3).

A different pattern was observed when patients were stratified according to neurological outcome. In patients with unfavorable outcome (CPC categories 3 to $5, n=102$ ), serum creatinine was significantly higher at admission (1.32 vs. $1.20 \mathrm{mg} /$ $\mathrm{dl}, P=0.039)$ when compared with patients with favorable neurological outcome $(n=69)$. While serum creatinine levels on average decreased in patients with good neurological outcome in the following two days, they increased in average in patients with unfavorable outcomes.

\section{Frequency of AKI stages 0 to 3}

A median urine output of $2000 \mathrm{~mL}$ (IQR 1300 to $2700 \mathrm{~mL}$, range 0 to $10,080 \mathrm{~mL}$ ) in the first 24 hours was found in the study population. There was no statistically significant difference between patients with good or unfavorable outcomes ( $P$ $=0.18$, Table 2). Oliguria (urine output $<500 \mathrm{~mL}$ ) was present in six patients with good outcome and in 11 with unfavorable outcome $(P=0.80)$. Renal replacement therapy was initiated

Table 2

\begin{tabular}{|c|c|c|c|c|}
\hline & $\begin{array}{l}\text { Study population } \\
(\mathrm{n}=171)\end{array}$ & $\begin{array}{c}\mathrm{CPC} 1+2 \\
(\mathrm{n}=69)\end{array}$ & $\begin{array}{c}\mathrm{CPC} 3+4+5 \\
(n=102)\end{array}$ & $P$ value \\
\hline Male gender & $135(78 \%)$ & $58(84 \%)$ & $76(74 \%)$ & 0.18 \\
\hline Age (years) & $63(53-72)$ & $60(52-69)$ & $65(54-74)$ & 0.13 \\
\hline $\mathrm{OHCA}$ & $145(86 \%)$ & $62(89 \%)$ & $83(81 \%)$ & 0.08 \\
\hline Bystander CPR & $46(27 \%)$ & $24(35 \%)$ & $22(22 \%)$ & 0.05 \\
\hline Cardiac cause of arrest & $142(83 \%)$ & $62(89 \%)$ & $79(78 \%)$ & 0.06 \\
\hline VF as initial rhythm & $111(65 \%)$ & $63(91 \%)$ & $48(47 \%)$ & 0.01 \\
\hline APACHE II score & $28(21-34)$ & $29(22-33)$ & $27(21-34)$ & 0.59 \\
\hline Urine output (I/24 h) & $2.0(1.3-2.7)$ & $2.0(1.5-2.7)$ & $1.9(1.2-2.7)$ & 0.18 \\
\hline Therapeutic hypothermia & $98(57 \%)$ & $52(75 \%)$ & $46(45 \%)$ & 0.002 \\
\hline NSE after 72 hours $(\mu \mathrm{g} / \mathrm{l})$ & $29.6(18.5-80.9)$ & $18.5(12.5-23.7)$ & $63(29-203)$ & $<0.001$ \\
\hline ICU length of stay (days) & $13(7-26)$ & $15(9-25)$ & $13(6-26)$ & 0.13 \\
\hline
\end{tabular}

Data are presented as medians (25th and 75th percentiles) or as absolute numbers (relative frequencies). APACHE: acute physiology and chronic health evaluation; CPC: cerebral performance category; CPR: cardiopulmonary resuscitation; ICU: intensive care unit; NSE: neuronspecific enolase; OHCA: out-of-hospital cardiac arrest; VF: ventricular fibrillation. 
Table 3

Course of serum creatinine over time in patients after cardiac arrest

\begin{tabular}{|c|c|c|c|c|}
\hline & $\begin{array}{c}\text { Study population } \\
(\mathrm{n}=171)\end{array}$ & $\begin{array}{c}\mathrm{CPC} 1+2 \\
(\mathrm{n}=69)\end{array}$ & $\begin{array}{c}\mathrm{CPC} 3+4+5 \\
(\mathrm{n}=102)\end{array}$ & $P$ value \\
\hline \multicolumn{5}{|c|}{ serum creatinine (mg/dl) } \\
\hline at admission & $1.24(1.01-1.65)$ & $1.20(0.94-1.49)$ & $1.32(1.08-1.68)$ & 0.039 \\
\hline after 24 hours & $1.12(0.74-1.87)$ & $0.79(0.60-1.49)$ & $1.35(0.96-2.06)$ & $<0.0001$ \\
\hline after 72 hours & $1.18(0.79-2.23)$ & $0.93(0.67-1.50)$ & $1.37(0.92-2.51)$ & 0.0174 \\
\hline at ICU discharge & $0.86(0.68-1.60)$ & $0.78(0.64-0.96)$ & $1.05(0.72-2.24)$ & 0.0003 \\
\hline$\Delta$ Crea24 & $-0.12(-0.35-0.30)$ & $-0.25(-0.51-0.02)$ & $0.02(-0.23-0.52)$ & $<0.0001$ \\
\hline$\Delta$ Crea72 & $-0.01(-0.33-0.68)$ & $-0.13(-0.38-0.24)$ & $0.04(-0.30-0.96)$ & 0.026 \\
\hline
\end{tabular}

Data are presented as medians (25th and 75th percentiles). The differences between patients with CPC 1 to 2 vs. CPC 3 to 5 were significant at every point of assessment. $\Delta$ Crea24: change in serum creatinine in the first 24 hours; $\Delta$ Crea72: change in serum creatinine in the first 72 hours; CPC: Cerebral Performance Category; ICU = intensive care unit.

in six patients with good outcome and in seven patients with unfavorable outcome $(P=0.88)$. Using serum creatinine levels at admission as baseline, AKI occurred more frequently in patients with unfavorable outcome. The difference compared with patients with good neurological outcome was statistically significant $(P=0.013$, Table 4).

\section{NSE serum levels and univariate and multivariate regression}

As expected, serum NSE values were significantly higher in patients with unfavorable outcomes $(63 \mu \mathrm{g} / \mathrm{L}$, IQR 29 to 203 $\mu \mathrm{g} / \mathrm{L}$, range 8.2 to $671 \mu \mathrm{g} / \mathrm{L})$ compared with patients with good neurological outcome $(18.5 \mu \mathrm{g} / \mathrm{L}$, IQR 12.5 to $23.7 \mu \mathrm{g} /$ $\mathrm{L}$, range 4.8 to $58.3 \mu \mathrm{g} / \mathrm{L}, P<0.001$, Table 2$)$.

Using simple regression we found that NSE levels correlated with $\Delta$ Crea24 $(r=0.24, P=0.002), \Delta$ Crea72 $(r=0.15, P=$ $0.049)$ and age $(r=-0.17, P=0.03)$, but not with Acute Physiology and Chronic Health Evaluation (APACHE)-II score, urine output and serum creatinine at admission (all $P>0.30$ ).

Table 4

Frequency of acute kidney injury stages 0 to 3

\begin{tabular}{lccc}
\hline & $\begin{array}{c}\text { Study population } \\
(\mathbf{n}=171)\end{array}$ & $\begin{array}{c}\text { CPC 1+2 } \\
(\mathbf{n = 6 9 )}\end{array}$ & $\begin{array}{c}\text { CPC 3 + 4+5 } \\
(\mathbf{n}=102)\end{array}$ \\
\hline AKI Stage 0 & $105(61 \%)$ & $50(72.5 \%)$ & $54(52.9 \%)$ \\
AKI Stage 1 & $28(16.3 \%)$ & $9(13 \%)$ & $19(18.6 \%)$ \\
AKI Stage 2 & $11(6.4 \%)$ & $3(4.3 \%)$ & $8(7.8 \%)$ \\
AKI Stage 3 & $28(16.3 \%)$ & $7(10.1 \%)$ & $21(20.6 \%)$ \\
\hline
\end{tabular}

Data are presented as absolute numbers (relative frequencies). AKI occurred significant more frequently in patients with unfavorable outcome (Chi-square test for trends, $P=0.013)$. AKI: acute kidney injury; CPC: cerebral performance category.
NSE serum levels were analyzed with a multivariate regression model including gender, age, APACHE II-score at admission, urine output in the first 24 hours and change in serum creatinine in the first 24 hours ( $\Delta$ Crea24) as independent factors. In this model, NSE levels were found to correlate with $\Delta$ Crea24 ( $r=0.24, P=0.0025)$ and age $(r=-0.17, P=0.048)$ independently of APACHE II-score $(r=-0.014, P=0.57)$, gender $(r=0.08, P=0.21)$ and urine output $(r=-0.07, P=0.90)$. The multiple correlation coefficient was 0.31 . The overall level of significance for the analysis of variance was $P=0.007$.

A similar pattern was found when performing the analysis with outcome as the dependent variable. In this model, outcome was found to correlate with $\Delta$ Crea24 $(r=0.21, P=0.0021)$ independently of APACHE II-score $(r=-0.006, P=0.21)$, gender $(r=0.18, P=0.05)$, age $(r=0.003, P=0.23)$ and urine output $(r=-0.000009, P=0.75)$. The multiple correlation coefficient was 0.29 . The overall level of significance for the analysis of variance was $P=0.011$.

\section{Risk stratification using $\Delta$ Crea24}

The prognostic value of $\Delta$ Crea24 in predicting favorable neurological outcome was evaluated using ROC analyses. The area under the curve was calculated with 0.69 (95\% confidence interval $(\mathrm{Cl}) 0.62$ to 0.76$)$. The value for prediction of good outcome with the highest accuracy was $\Delta$ Crea24 less than $-0.19 \mathrm{mg} / \mathrm{dl}$. When this threshold was applied, good outcomes could be predicted with a sensitivity of $63 \%$ and a specificity of $71 \%$ (positive likelihood ratio (LR) 1.9, negative LR 0.4). Moreover, we found that the relative risk (RR) for unfavorable neurological outcome (CPC 3 to 5 ) was 2.1 (95\% Cl 1.5 to 3.0) in cases of unchanged or positive $\Delta$ Crea24 ( $P=$ $0.0001)$. When $\Delta$ Crea24 declined by more than $0.2 \mathrm{mg} / \mathrm{dl}$, the RR for the occurrence of unfavorable neurological outcome was $0.46(95 \% \mathrm{Cl} 0.32$ to $0.68, P=0.0001)$. The odds ratio was 3.81 (95\% Cl 1.98 to 7.33$), P=0.0001$ or $0.27(95 \% \mathrm{Cl}$ 
0.14 to $0.51, P=0.0001)$, respectively. For interval LRs for $\Delta$ Crea24, please refer to Table 5 .

\section{Discussion}

We demonstrate that $\mathrm{AKI}$ is common in patients after cardiac arrest when the new AKI criteria are applied. Patients with unfavorable neurological outcome are affected significantly more frequently. Furthermore, we found a direct significant association between AKI and serum levels of NSE as a marker of hypoxic brain damage.

$\mathrm{AKI}$ is a known complication after cardiac arrest although different definitions of 'renal failure' in the past have made comparisons difficult [16]. In a recent investigation some pre-arrest factors including history of hypertension, chronic heart failure and chronic renal insufficiency could be identified as risk factors for renal failure after cardiac arrest and an association between acute renal failure and epinephrine dosage during cardiopulmonary resuscitation was found [17]. This may indicate that the extent of hypoxia/ischemia may also play a role in the development of AKI. In fact, acute renal failure could be induced by cardiac arrest in a mouse model [18]. This finding is in line with our data suggesting an association between hypoxia and the development of AKI after cardiac arrest. Furthermore, our data indicate that early changes in serum creatinine might help to predict outcome in these patients: while a decline of serum creatinine levels of $0.2 \mathrm{mg} / \mathrm{dl}$ or more in the first 24 hours after cardiac arrest may indicate good prognosis, constant or even increasing levels seem to predict unfavorable outcome.

A number of limitations to our analysis require careful consideration. First, our data were obtained in a single-center cohort and thus require validation studies before clinical application. Second, the detection of differences in creatinine levels of 0.2 $\mathrm{mg} / \mathrm{dl}$ is sophisticated with regard to the precision of the test. Furthermore, data about kidney function prior to cardiac arrest were not available. This may be an important point because our data suggest a temporary rise in serum creatinine during the first hours after successful cardio-pulmonary resuscitation. Although an early rise in serum creatinine following cardiac arrest was also found in previous observations, the reason for this phenomenon remains unknown [19]. On the whole, it is not clear if such early changes in serum creatinine indeed represent real alterations in glomerular filtration rate. Thus, serum creatinine at admission is not a reliable measure for chronic kidney function in these patients. As we have used the serum creatinine at admission as the baseline for defining AKI, the incidence of acute kidney disease may be underestimated in our cohort.

Moreover, creatine release from skeletal muscles during cardiopulmonary resuscitation may theoretically influence the course of serum creatinine levels. Although we are unable to rule out an effect of muscular release of creatine with certainty, serum creatine kinase levels were not found to correlate with serum creatinine levels or with changes in serum creatinine levels at baseline and over time ( $P>0.5$ for all comparisons). Moreover, serum creatine kinase was not found to discriminate between favorable and unfavorable outcome (data not shown). Kidney function may be also affected by treatment with therapeutic hypothermia. Although recent investigations did not detect differences in the incidence of acute renal failure under hypothermia, transient effects on renal function cannot be fully excluded [20].

Concerning neurological outcome, we only present CPC scores at ICU discharge. Although some evidence indicates that there are only minor changes regarding neurological outcome after ICU discharge [21], long-term follow up may provide more insight into this important endpoint. Moreover, one should keep in mind that classification as CPC 5 may reflect two different clinical situations: Patients dying in a comatose state after therapy withdrawal and patients dying from other complications after regaining consciousness. Nevertheless, although the neurological situation seems completely different, from the patient's point of view CPC 5 is an important outcome variable independent of the cause of death. In addition,

Table 5

\begin{tabular}{|c|c|c|c|c|}
\hline$\Delta$ Crea24 (mg/dl) & $\begin{array}{c}\mathrm{CPC} 1+2 \\
(n=69)\end{array}$ & $\begin{array}{c}\text { CPC } 3+4+5 \\
(n=102)\end{array}$ & Likelihood ratio & $95 \%$ confidence interval \\
\hline$<-0.4$ & 27 & 10 & 0.251 & 0.130 to 0.484 \\
\hline$-0.4--0.2$ & 15 & 20 & 0.902 & 0.497 to 1.636 \\
\hline$-0.2-0.0$ & 8 & 19 & 1.607 & 0.746 to 3.461 \\
\hline $0.0-0.2$ & 8 & 17 & 1.437 & 0.657 to 3.145 \\
\hline $0.2-0.4$ & 3 & 8 & 1.804 & 0.496 to 6.562 \\
\hline$>0.4$ & 8 & 28 & 2.368 & 1.148 to 4.883 \\
\hline
\end{tabular}

Interval likelihood ratios with $95 \%$ confidence interval for $\Delta$ Crea24. The number of patients with unfavorable vs. favorable outcome is given for respective $\Delta$ Crea24 intervals. $\Delta$ Crea24: change in serum creatinine in the first 24 hours; CPC: Cerebral Performance Category. 
there is good evidence that the majority of patients after cardiac arrest die after therapy withdrawal [22].

Importantly, our data should not be used to predict outcome in patients after cardiac arrest. Obviously, when predicting neurological outcome one should focus on the brain, not the kidney, and reliable multimodal approaches are available for this purpose [23]. Nevertheless, the demonstrated relation between the kidney and the brain may help to identify patients at a high risk of an unfavorable outcome. Theoretically, this may have implications for ICU care in the future. In patients with sepsis, convincing data demonstrate that early identification and therapy using an early goal-directed therapeutic approach with fluids and vasopressor support improves organ function and outcome [24]. Although somewhat speculative, one might argue that these rather simple approaches may also be effective in patients after cardiac arrest via improvement of both cerebral and kidney function.

Moreover, there may be another conclusion which may be drawn from our data. Nearly half of the patients with severe hypoxic brain damage after cardiac arrest did not develop AKI despite profound global ischemia. This result is in marked contrast to the situation typically found in severe shock and multiple-organ failure where acute renal failure is a common condition but relevant encephalopathy a comparably rare event. In this light, our findings support the hypothesis that 'simple' hypoperfusion may be only one piece in the puzzle of the complex pathophysiology of AKI. In fact, mounting evidence from animal models indicate that $\mathrm{AKI}$ may develop without renal ischemia in sepsis [25-27]. As a consequence, critical care physicians should be once more careful when extrapolating results obtained from animal models to the clinical situation of our patients. One might speculate that eventually we will need to differentiate 'high flow' from 'low flow' AKI and accordingly apply different treatment strategies in the future.

\section{Conclusions}

In summary, we demonstrate that AKI occurs in nearly $50 \%$ of patients after cardiac arrest when the new AKI criteria are applied. Patients with unfavorable neurological outcome are affected more frequently. Furthermore, we found a significant association between AKI and serum levels of NSE as a marker of hypoxic brain damage. Our data indicate that changes in serum creatinine might be an early predictor of outcome in these patients and that a decrease of serum creatinine in the first 24 hours of more than $0.2 \mathrm{mg} / \mathrm{dl}$ may be a sign of good prognosis, whereas constant or even increasing serum creatinine levels indicate unfavorable outcomes.

\section{Competing interests}

The authors declare that they have no competing interests.

\section{Key messages}

- $\mathrm{AKI}$ is frequent after cardiac arrest - approximately $50 \%$ of cardiac arrest patients may be affected.

- Development of $\mathrm{AKI}$ is associated with unfavorable neurological outcome and increased mortality in patients after cardiac arrest.

- Whereas a decline in serum creatinine levels in the first 24 hours may indicate favorable prognosis, constantly elevated or even further increased serum creatinine levels indicate an unfavorable outcome after cardiac arrest.

\section{Authors' contributions}

$\mathrm{DH}, \mathrm{CS}, \mathrm{SvH}$ and JCS designed and supervised the study from data acquisition to data analysis. AJ participated in the design of the study, revised the manuscript for important intellectual content and helped to draft the manuscript. All authors have read and approved the final version of the manuscript.

\section{References}

1. Uchino S, Doig GS, Bellomo R, Morimatsu H, Morgera S, Schetz M, Tan I, Bouman C, Nacedo E, Gibney N, Tolwani A, Ronco C, Kellum JA: Diuretics and mortality in acute renal failure. Crit Care Med 2004, 32:1669-1677.

2. Chertow GM, Burdick E, Honour M, Bonventre JV, Bates DW: Acute kidney injury, mortality, length of stay, and costs in hospitalized patients. J Am Soc Nephrol 2005, 16:3365-3370.

3. Mehta RL, Kellum JA, Shah SV, Molitoris BA, Ronco C, Warnock DG, Levin A: Acute Kidney Injury Network: report of an initiative to improve outcomes in acute kidney injury. Crit Care 2007, 11:R31.

4. Lopes JA, Jorge S, Resina C, Santos C, Pereira A, Neves J, Antunes F, Prata MM: Acute kidney injury in patients with sepsis: a contemporary analysis. Int J Infect Dis 2009, 13:176-181.

5. Abelha FJ, Botelho M, Fernandes V, Barros H: Determinants of postoperative acute kidney injury. Crit Care 2009, 13:R79.

6. Ozcakar ZB, Yalcinkaya F, Altas B, Ergun H, Kendirli T, Ates C, Elhan AH, Ekim M: Application of the new classification criteria of the Acute Kidney Injury Network: a pilot study in a pediatric population. Pediatr Nephrol 2009, 24:1379-1384.

7. Cruz D, Ricci Z, Ronco C: Clinical review: RIFLE and AKIN - time for reappraisal. Crit Care 2009, 13:211.

8. Heyman SN, Lieberthal W, Rogiers P, Bonventre JV: Animal models of acute tubular necrosis. Curr Opin Crit Care 2002, 8:526-534.

9. Lameire N, Van BW, Vanholder R: Acute renal failure. Lancet 2005, 365:417-430.

10. Bulut S, Aengevaeren WR, Luijten HJ, Verheugt FW: Successful out-of-hospital cardiopulmonary resuscitation: what is the optimal in-hospital treatment strategy? Resuscitation 2000, 47:155-161.

11. Meynaar IA, Oudemans-van Straaten HM, van der WJ, Verlooy $P$, Slaats EH, Bosman RJ, Spoel Jl van der, Zandstra DF: Serum neuron-specific enolase predicts outcome in post-anoxic coma: a prospective cohort study. Intensive Care Med 2003, 29:189-195.

12. Reisinger $J$, Hollinger $K$, Lang $W$, Steiner $C$, Winter $T$, Zeindlhofer E, Mori M, Schiller A, Lindorfer A, Wiesinger K, Siostrzonek P: Prediction of neurological outcome after cardiopulmonary resuscitation by serial determination of serum neuron-specific enolase. Eur Heart J 2007, 28:52-58.

13. Almaraz AC, Bobrow BJ, Wingerchuk DM, Wellik KE, Demaerschalk BM: Serum neuron specific enolase to predict neurological outcome after cardiopulmonary resuscitation: a critically appraised topic. Neurologist 2009, 15:44-48.

14. Levey AS, Bosch JP, Lewis JB, Greene T, Rogers N, Roth D: A more accurate method to estimate glomerular filtration rate from serum creatinine: a new prediction equation. Modification 
of Diet in Renal Disease Study Group. Ann Intern Med 1999, 130:461-470.

15. Jennett $B$, Bond $M$ : Assessment of outcome after severe brain damage. Lancet 1975, 1:480-484.

16. Mattana J, Singhal PC: Prevalence and determinants of acute renal failure following cardiopulmonary resuscitation. Arch Intern Med 1993, 153:235-239.

17. Domanovits $H$, Schillinger M, Mullner M, Thoennissen J, Sterz F, Zeiner A, Druml W: Acute renal failure after successful cardiopulmonary resuscitation. Intensive Care Med 2001, 27:1194-1199.

18. Burne-Taney MJ, Kofler J, Yokota N, Weisfeldt M, Traystman RJ, $\mathrm{Rabb} \mathrm{H}$ : Acute renal failure after whole body ischemia is characterized by inflammation and T cell-mediated injury. Am J Physiol Renal Physiol 2003, 285:F87-F94.

19. Domanovits $H$, Mullner $M$, Sterz $F$, Schillinger $M$, Klosch $C$, Paulis M, Hirschl MM, Laggner AN: Impairment of renal function in patients resuscitated from cardiac arrest: frequency, determinants and impact on outcome. Wien Klin Wochenschr 2000, 112:157-161.

20. Zeiner A, Sunder-Plassmann G, Sterz F, Holzer M, Losert $H$, Laggner AN, Mullner M: The effect of mild therapeutic hypothermia on renal function after cardiopulmonary resuscitation in men. Resuscitation 2004, 60:253-261.

21. Nielsen N, Hovdenes J, Nilsson F, Rubertsson S, Stammet $P$, Sunde K, Valsson F, Wanscher M, Friberg H: Outcome, timing and adverse events in therapeutic hypothermia after out-ofhospital cardiac arrest. Acta Anaesthesiol Scand 2009, 53:926-934.

22. Geocadin RG, Buitrago MM, Torbey MT, Chandra-Strobos N, Williams MA, Kaplan PW: Neurologic prognosis and withdrawal of life support after resuscitation from cardiac arrest. Neurology 2006, 67:105-108

23. Wijdicks EF, Hijdra A, Young GB, Bassetti CL, Wiebe S: Practice parameter: prediction of outcome in comatose survivors after cardiopulmonary resuscitation (an evidence-based review): report of the Quality Standards Subcommittee of the American Academy of Neurology. Neurology 2006, 67:203-210.

24. Rivers E, Nguyen B, Havstad S, Ressler J, Muzzin A, Knoblich B, Peterson E, Tomlanovich M: Early goal-directed therapy in the treatment of severe sepsis and septic shock. N Engl J Med 2001, 345:1368-1377.

25. Bellomo R, Wan L, Langenberg C, May C: Septic acute kidney injury: new concepts. Nephron Exp Nephro/ 2008, 109:e95-100.

26. Wan L, Bagshaw SM, Langenberg C, Saotome T, May C, Bellomo R: Pathophysiology of septic acute kidney injury: what do we really know? Crit Care Med 2008, 36:S198-S203.

27. Langenberg C, Wan L, Egi M, May CN, Bellomo R: Renal blood flow and function during recovery from experimental septic acute kidney injury. Intensive Care Med 2007, 33:1614-1618. 